\title{
ELECTROMAGNETIC FIELD MODE IN FIREBALL FORMATION
}

\author{
N. Kondo, M. Kamogawa, H. Ofuruton* and Y. H. Ohtsuki \\ Department of Physics, Waseda University \\ 4-1, Okubo 3, Shinjuku-ku, Tokyo 169, Japan \\ *Tokyo Metropolitan College of Aeronautical Engineering \\ 52-1, Minamisenju 8, Arakawa-ku, Tokyo 116, Japan
}

\begin{abstract}
It is generally known that ball lightnings exist in nature. Ohtsuki and Ofuruton $(1991 ; 1994)$ were successful in producing plasma fireballs like ball lightnings in laboratory by using microwave interference in a cavity. According to Kapitza(1955)'s theory, ball lightnings appear at high intensity regions of electromagnetic wave interference. However, the experimental fireballs produced by Ohtsuki and Ofuruton were able to appear at regions where electromagnetic field of high intensity did not exist theoretically. We used a new method in measuring the electromagnetic field mode by using thermal paper and made sure of a possibility that electromagnetic fields became high around fireballs.
\end{abstract}

\section{Introduction}

There are a lot of eyewitness reports of luminous spherical objects under the condition of thunderstorm(Singer 1971; Barry, 1980; Smirnov, 1993). The objects are so-called ball lightnings. Ball lightnings are very rare phenomena but they have been studied by many scientists experimentally and theoretically(Ohtsuki, 1988; Smirnov, 1993).

Kapitza(1955) proposed a theory of ball lightning. He predicted that ball lightnings were produced at the high intensity regions of radio waves caused by thunderstorms. His theory suggested that ball lightnings should appear at the antinodes of the standing wave by electromagnetic wave interference.

Ohtsuki and Ofuruton(1991; 1994) produced plasma fireballs like ball lightnings in laboratory by using microwave interference in a cavity filled with atmospheric air. Their experiment was motivated by Kapitza's theory. In the experiment, the fireballs tended to go upward when they appeared. And the fireballs were often stable on the upper wall of the cavity. The property that the fireballs went upward is explained by their buoyancy or convection. But, when the fireballs went upward, they should disappear immediately by deexcitation, because the fireballs could not exist on the metallic wall where there was the node of standing wave and the intensity of the microwave was very low theoretically. However, the fireballs did not disappear, went upward and stayed in the upper part of the cavity. It is suggested that the high field region moves together with a fireball when the fireball go upward.

In this paper, we report on our new experiment to measure the electric field mode in the cavity and the reason why fireballs can go upward and be stable without their extinction.

\section{Experiment}

A schematic diagram of the apparatus of this experiment is shown in Fig.1. We used a $2.45 \mathrm{GHz}, 5 \mathrm{~kW}$ (maximum power) microwave oscillator(magnetron) operating continuously. The electromagnetic mode was $H_{01}$ mode in the experiment. The microwave was guided through a rectangular waveguide $(55 \mathrm{~mm} \times 109 \mathrm{~mm})$ straight to a 
cavity made of aluminum punched plates. The cavity was $161 \mathrm{~mm}$ in diameter and $370 \mathrm{~mm}$ in length.

We used thermal paper to observe the electric field. This thermal paper is ordinarily used for thermal printers. The color of the thermal paper changes from white to black at high temperature regions. Electric field of high intensity also can change the color of paper. Thus, we were able to qualitatively measure the electric field in the cavity by using the paper.

In this experiment, we used an aluminum ball instead of a plasma fireball. We had two reasons for using the aluminum ball. The first reason was that the thermal paper would be burned if a plasma fireball touched it. The second reason was that the plasma fireball was electrically conductive as well as metal. The diameter of the aluminum ball was $2 \mathrm{~cm}$ which corresponded to that of a plasma fireball in our previous experiment.

Figure 2 shows an arrangement of the thermal paper and the aluminum ball in the cavity. We set the thermal paper in the cavity and put the aluminum ball on various places. And we operated the magnetron and measured the electric field.

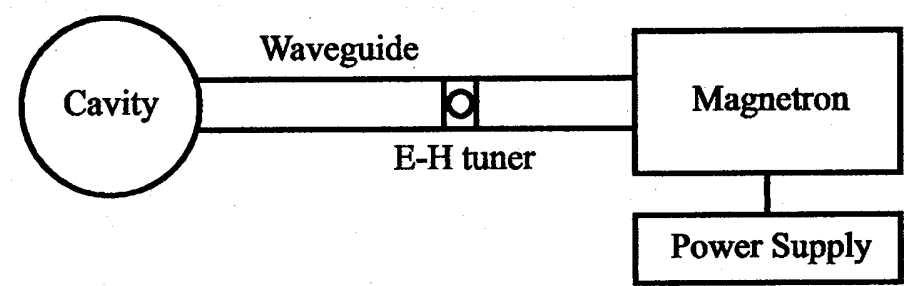

Fig.1 Schematic diagram of the apparatus.

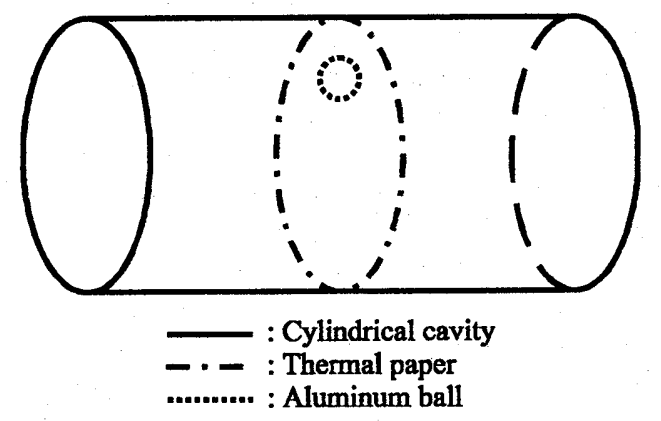

Fig. 2 Arrangement of the thermal paper in the cavity.

\section{Results and Discussion}

First, as a test, we set the thermal paper on the edge of the waveguide and measured the electric field in the waveguide. The result is shown in Fig.3. The color of thermal paper changed into black like a pillar at the center of the waveguide because the electromagnetic field mode was $\mathrm{H}_{01}$. The result shows that the thermal paper is very useful in measuring the electric field mode. 


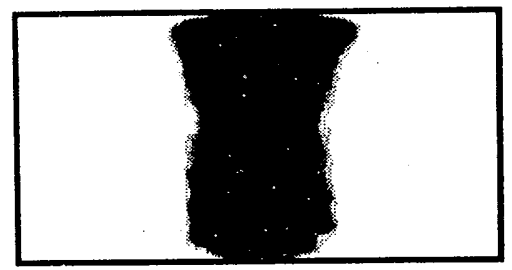

Fig. 3 Electric field mode in the waveguide.

The rectangle is the cross section of the waveguide.

Power of the microwave: $5 \mathrm{~kW}$. Irradiation time: $20 \mathrm{sec}$.

In our previous experiment in 1991, we mainly used a handmade cavity whose shape was approximately cylindrical. It was difficult for us to produce a fireball by using a manufactured cavity which was ideally cylindrical. The reason was unknown. Therefore, we measured the electromagnetic field in two cases of a handmade cavity and a manufactured cavity.

Figure 4(a) shows the field mode of the manufactured cavity to compare with that of the handmade cavity. The shape of the black part is like a cylinder. This result corresponds to the theoretical and ideal mode. The manufactured cavity was so good cavity that the paper was blackened enough by the low power microwave in the short irradiation time.

The electric field mode of Fig.4(b) of the handmade cavity is very different from the theoretical and ideal mode like Fig.4(a). In the handmade cavity, it was easy to produce a plasma fireball. We think that the shape of the handmade cavity is not so precise that the mode in the cavity is irregular. But we could not decide that the reason of effectiveness of the handmade cavity is in its imperfect shape.

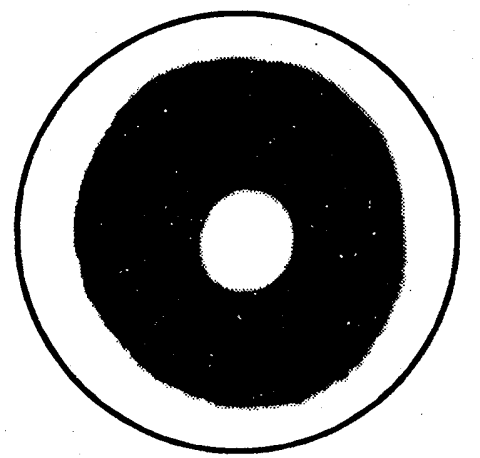

(a)

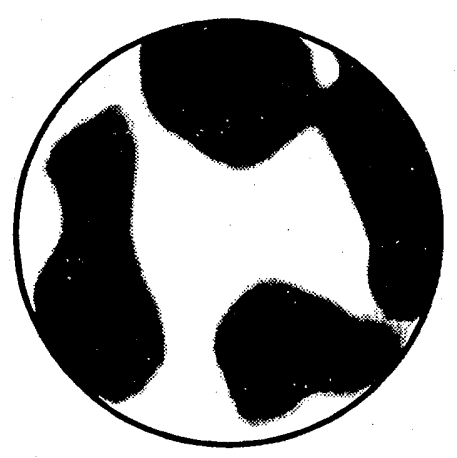

(b)

Fig.4 Electric field mode in the manufactured cavity(a) and in the handmade cavity(b). Each circle is the cross section of each cavity.

(a) Power of the microwave: $1 \mathrm{~kW}$. Irradiation time: $5 \mathrm{sec}$.

(b) Power of the microwave: $2 \mathrm{~kW}$. Irradiation time: $40 \mathrm{sec}$. 
Next, we put an aluminum ball in the center or the upper part of the cavity and measured the electric field. In the case that the aluminum ball was at the center of the cavity, the result is shown in Fig.5(a). In another case that the aluminum ball was in the upper part of the cavity, the result is shown in Fig.5(b). The figures indicate that the electric field is high around the aluminum ball, which simulates the fireball. We do not consider these blackened parts caused by thermal conduction from the aluminum ball, because there was a little space $(\sim 1 \mathrm{~mm})$ between the aluminum ball and each pieces of the paper, and the aluminum ball did not touch the paper. Although we did not measure the temperature of the aluminum ball, it was heated by the induction heating. It became so hot that we could not touch it by our bare hands.

The irradiation time of Fig.5(b) was shorter than that of Fig.5(a) because we avoided burning the paper. Consequently, color of Fig.5(b) (especially the lower part) is lighter than that of Fig.5(a). same conditions.

The color patterns like Fig.5(a) and (b) were reproducible under the

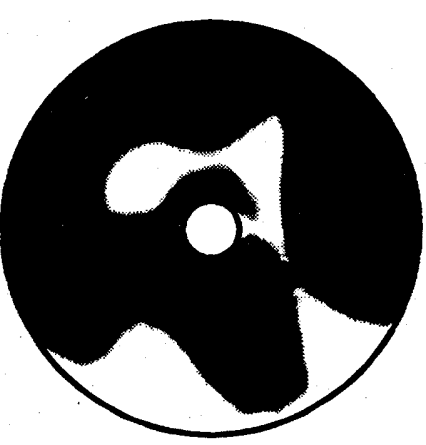

(a)

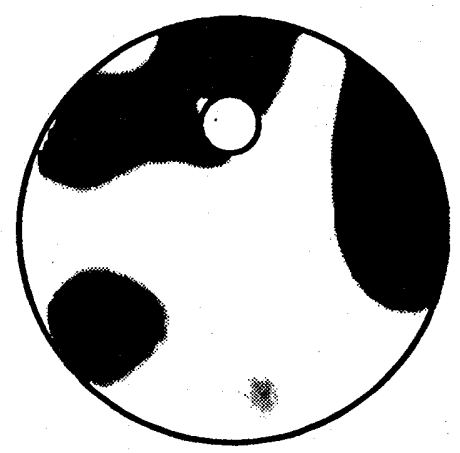

(b)

Fig. 5 Electric field mode in the handmade cavity with an aluminum ball at the center of the cavity(a) and at the upper part of the cavity(b). Each large circle is the cross section of the cavity. Each small circle is the cross section of the aluminum ball.

(a) Power of the microwave: $2 \mathrm{~kW}$. Irradiation time: $30 \mathrm{sec}$.

(b) Power of the microwave: $2 \mathrm{~kW}$. Irradiation time: $20 \mathrm{sec}$.

\section{Conclusions}

We proposed a new simple method using thermal paper to qualitatively observe high intensity electric field. The method was very useful to us for investigating the characteristics of our cavities because color patterns in thermal papers became the same under the same conditions. We measured electric field in our cavity by using the method. The electromagnetic field mode in our handmade cavity was very different from the ideal mode. The electric field was high around the aluminum ball which existed in the region where the electric field was low in the ideal mode. Thus, it was indicated that the electric field mode was modified by the aluminum ball. Therefore, it is suggested that the high intensity region of the electric field moves together with the fireball, resultantly the electric field is always high around the fireball. And then, the electric field is high near the upper wall of the cavity when the fireball goes upward although the electric field is theoretically low near the metallic wall of the cavity if the fireball is absent. 
Acknowledgment. The authors would like to thank Mr. Y. Tokumura and the members of Ohtsuki laboratory for their useful discussions and help.

\section{References}

Barry, J. D., Ball Lightning and Bead Lightning, Plenum Press, New York, pp.298, 1980. Kapitza, P. L. , On the nature of ball-lightning, Dokl. Akad. Nauk SSSR, 101, 245-248, 1955(in Russian) (English translation: Collected Papers of Kapitza, Vol.2, ed. by D. Ter Haar, Pergamon Press, London, 776-780, 1965).

Ohtsuki, Y. H. ed., Science of Ball Lightning(Fire Ball), World Scientific Publishing, Singapore, pp.339, 1988.

Ohtsuki, Y. H., and H. Ofuruton, Plasma fireballs formed by microwave interference in air, Nature, 350, 139-141, 1991.

Ohtsuki, Y. H. and H. Ofuruton, Nature of fireballs produced by microwave interference, Dusty and Dirty Plasmas, Noise, and Chaos in Space and in the Laboratory ed. by H. Kikuchi, Plenum Press, New York, 577-579, 1994.

Singer, S., The Nature of Ball Lightning, Plenum Press, New York, pp.169, 1971.

Smirnov, B.M., Physics of ball lightning, Physics Reports, 224, 151-236, 1993.

(Received September 11, 1997; revised December 29, 1997; accepted January 5, 1998.) 